\title{
A formalization of one of the main claims of "Cost-effectiveness analysis of pharmacogenomics-guided clopidogrel treatment in Spanish patients undergoing percutaneous coronary intervention" by Fragoulakis et al. 2019
}

\author{
George P. Patrinos \\ School of Health Sciences, Department of Pharmacy, University of Patras, Patras, Greece \\ E-mail: gpatrinos@upatras.gr; ORCID: https://orcid.org/0000-0002-0519-7776 \\ Editor: Cristina-Iulia Bucur (https://orcid.org/0000-0002-7114-6459) \\ Review comments from: Tobias Kuhn (https://orcid.org/0000-0002-1267-0234); Davide Ceolin \\ (https://orcid.org/0000-0002-3357-9130); Margherita Martorana (https://orcid.org/0000-0001-8004-0464); Cristina-Iulia \\ Bucur (https://orcid.org/0000-0002-7114-6459)
}

Received 5 July 2021

Accepted 17 November 2021

Abstract. Fragoulakis et al. claimed in previous work that pharmacogenomics-guided clopidogrel strategy represents a costeffective treatment for patients undergoing PCI. We present here a formalization of that claim, stating that all things of class "pharmacogenomics guided clopidogrel therapy" that are in the context of a thing of class "patient undergoing PCI" generally have a relation of type "enables" to a thing of class "cost-effective treatment" in the same context.

Keywords: Patient undergoing PCI, pharmacogenomics-guided clopidogrel therapy, cost-effective treatment

\section{Introduction}

Fragoulakis et al. [2] state that "Our data show that pharmacogenomics-guided clopidogrel treatment strategy may represent a cost-effective choice compared with non-pharmacogenomics-guided strategy for patients undergoing PCI.". We present here a formalization of the main scientific claim from this quote by using a semantic template called the super-pattern [1].

\footnotetext{
${ }^{1}$ As RDF/nanopublication: http://purl.org/np/RAn15vsPJEVdJvjNKtBPo_oadtjeP9oc3Si-69FiJ4poQ 


\section{Formalization}

Our formalization looks as follows:

CONTEXT-CLASS ("in the context of all..."):

SUBJECT-CLASS ("things of type..."):

QUALIFIER:

RELATION-TYPE ("have a relation of type..."):

OBJECT-CLASS ("to things of type..."): patient undergoing PCI

pharmacogenomics guided clopidogrel therapy

generally

enables

cost-effective treatment

In the context class we use a new minted class "patient undergoing PCI" that is a subclass of "patient" (Q181600) from Wikidata and is related to the class "percutaneous coronary intervention" (Q2008344) from Wikidata. In the subject class, we use a new minted class "pharmacogenomics guided clopidogrel therapy" that is a subclass of "treatment" (Q179661) from Wikidata and is related to the class "pharmacogenomics" (Q1152227) and "clopidogrel" (Q410237) from Wikidata. In the object class we minted a new class "cost-effective treatment" that is a subclass of "treatment" (Q179661) from Wikidata and is related to the class "cost-effectiveness analysis" (Q1754768) from Wikidata.

\section{RDF code}

This is our formalization as a nanopublication in TriG format:

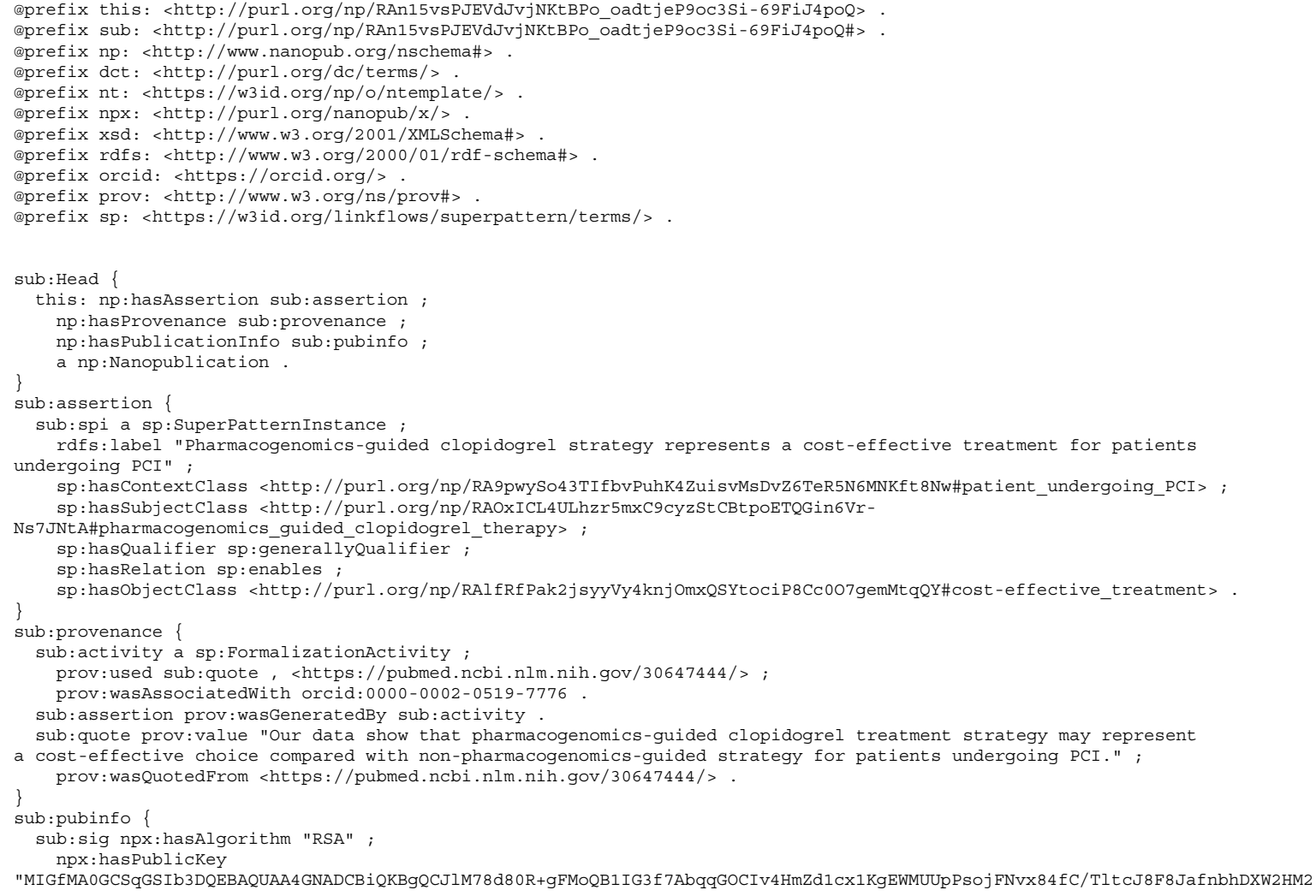




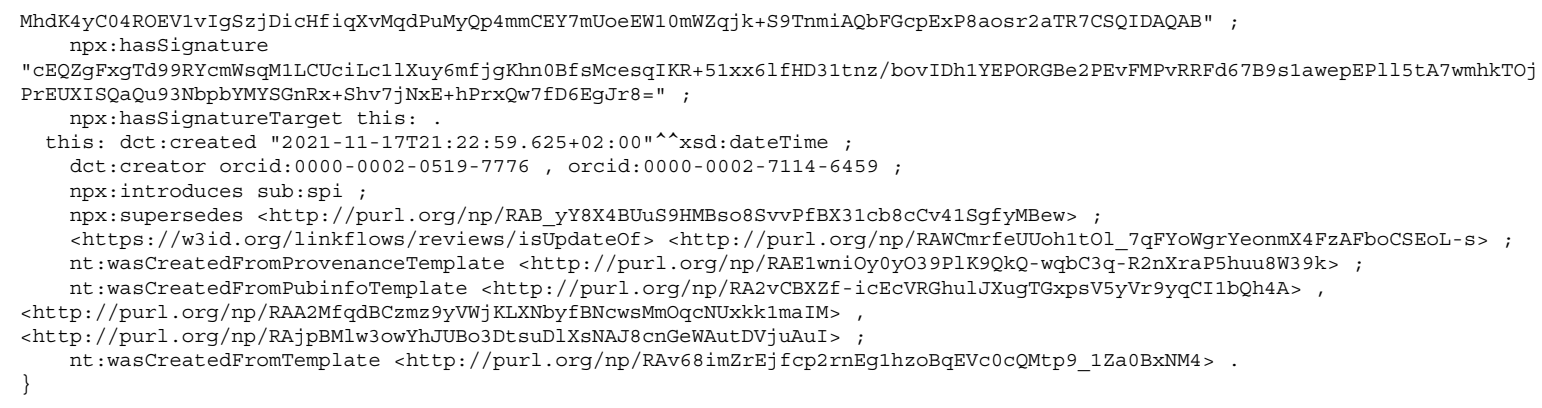

\section{The following nanopublications introduce the newly minted classes in TriG format.} This is the class definition of "patient undergoing PCI":

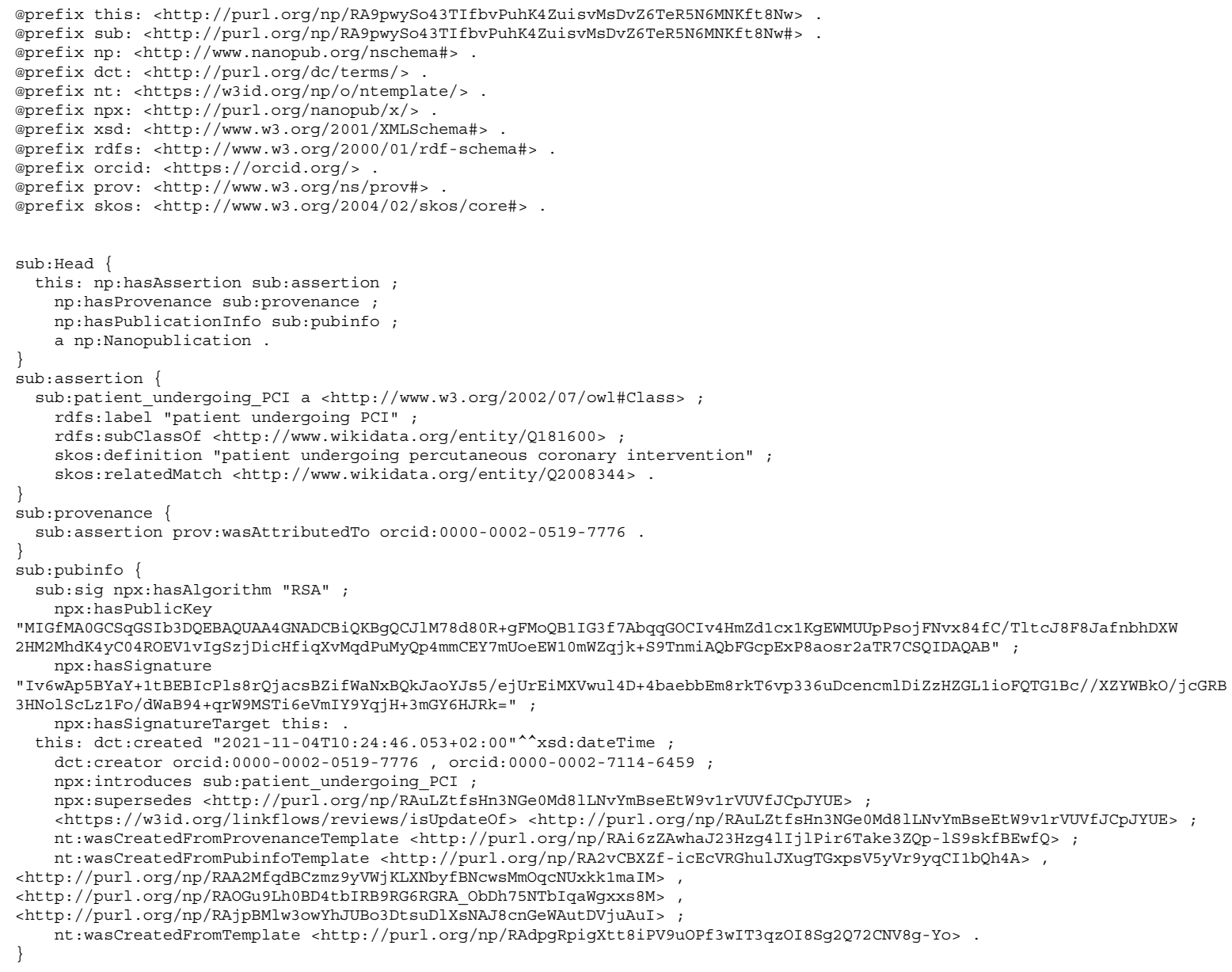

\section{This is the class definition of "pharmacogenomics guided clopidogrel therapy":}

@prefix this: <http://purl.org/np/RAOxICL4ULhzr5mxC9CyzStCBtpoETOGin6Vr-Ns7JNtA> @prefix sub: <http://purl.org/np/RAOxICL4ULhzr5mxC9CyzStCBtpoETOGin6Vr-Ns7JNtA\#> @prefix np: <http://www. nanopub.org/nschema\#> . @prefix dct: <http://purl.org/dc/terms/> @prefix nt: <https://w3id.org/np/o/ntemplate/> @prefix npx: <http://purl.org/nanopub/x/> 


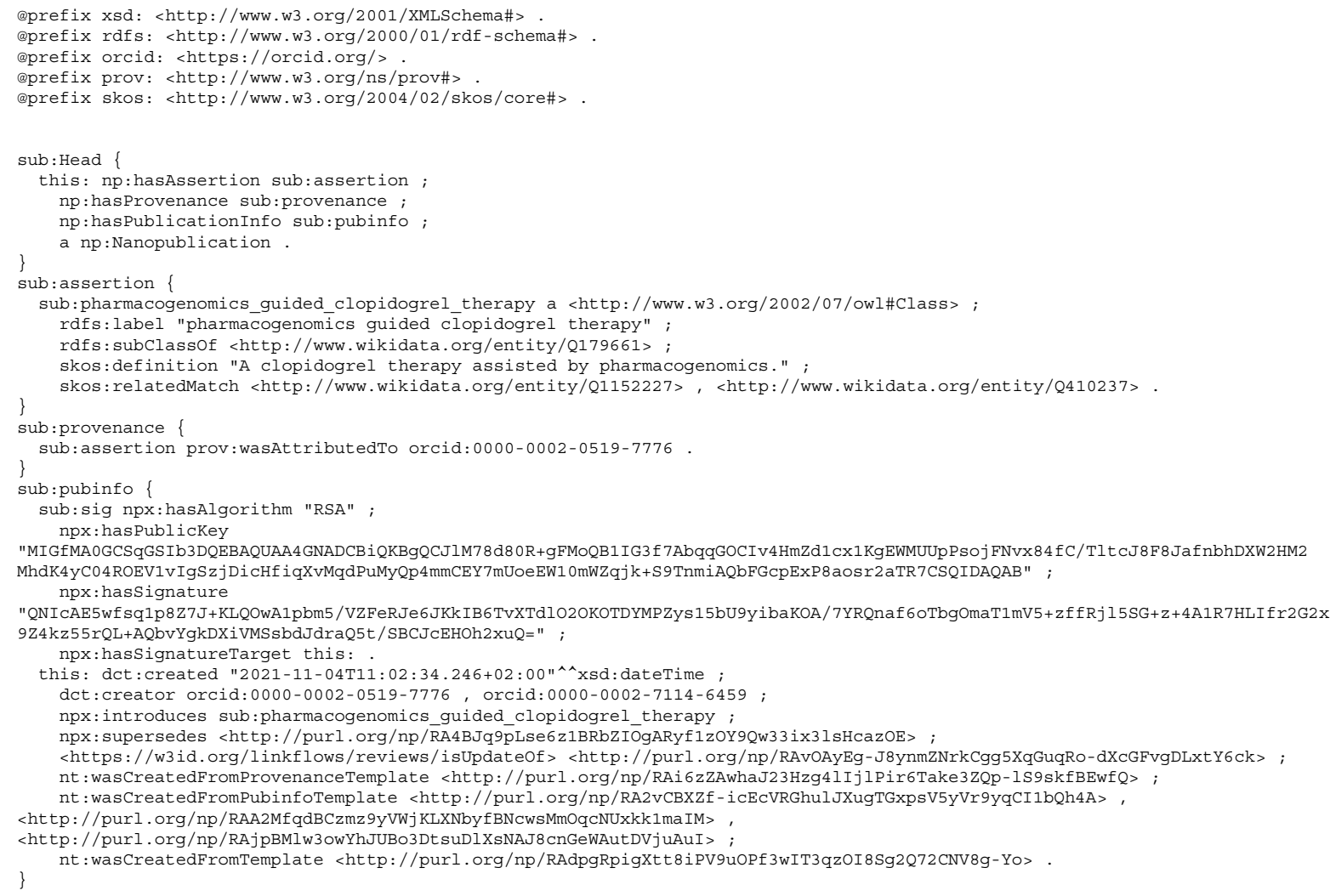

\section{This is the class definition of "cost-effective treatment":}

@prefix this: <http://purl.org/np/RAlfRfPak2j syYVy4knjOmxQSYtociP8Cc007gemMtqQY> @prefix sub: <http://purl.org/np/RAlfRfPak2j syYVy4knj OmxQSYtociP8Cc007gemMtqQY\#> . @prefix np: <http://www. nanopub.org/nschema\#>

@prefix dct: <http://purl.org/dc/terms/>

@prefix nt: <https://w3id.org/np/o/ntemplate/>

@prefix npx: <http://purl.org/nanopub/x/>

@prefix xsd: <http://www.w3.org/2001/xMLSchema\#>

@prefix rdfs: <http://www.w3.org/2000/01/rdf-schema\#> .

@prefix orcid: <https://orcid.org/>

@prefix prov: <http://www.w3.org/ns/prov\#>

@prefix skos: <http://www.w3.org/2004/02/skos/core\#> .

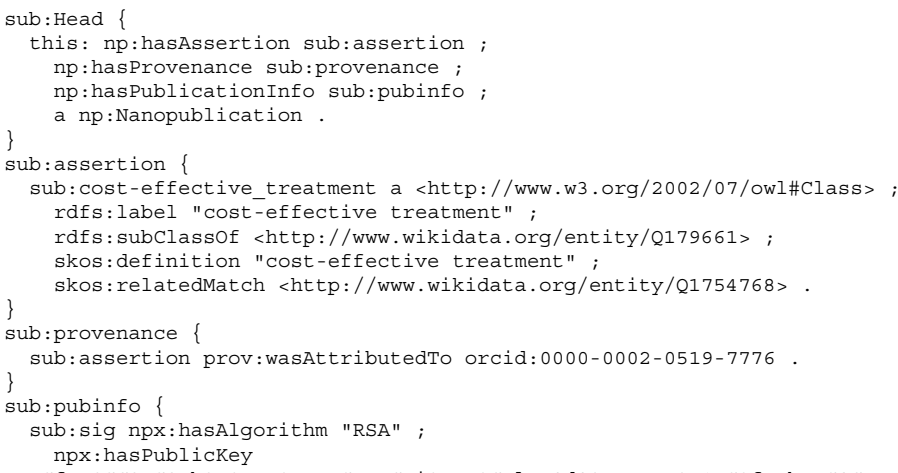

"MIGFMAOGCSqGSIb3DQEBAQUAA4 GNADCBi QKBgQCJ1M78d80R+gFMoQB1 IG3f 7AbqqGOCIV4HmZd1 cx1KgEWMUUpPsoj FNvx84fC/TltcJ8F8JafnbhDXW2HM2Mhd K4YC04ROEV1vIgSzjDicHfiqXVMqdPuMYQp4mmCEY7mUoeEW10mWZqjk+S9TnmiAQbFGCPEXP8aosr2aTR7CSQIDAQAB" ; npx: hassignature

"f+XJLOVxSGhXnuQYxbgd+cAlljMEdNppq011jalPaJlnUREFKwRB5A9mhsvEuV1TWGzHf zilwEL8FiguHgg7YUTJlbAlpb8qA98/MFkWtXeYzIj wOgmuFUrSIBQF0 


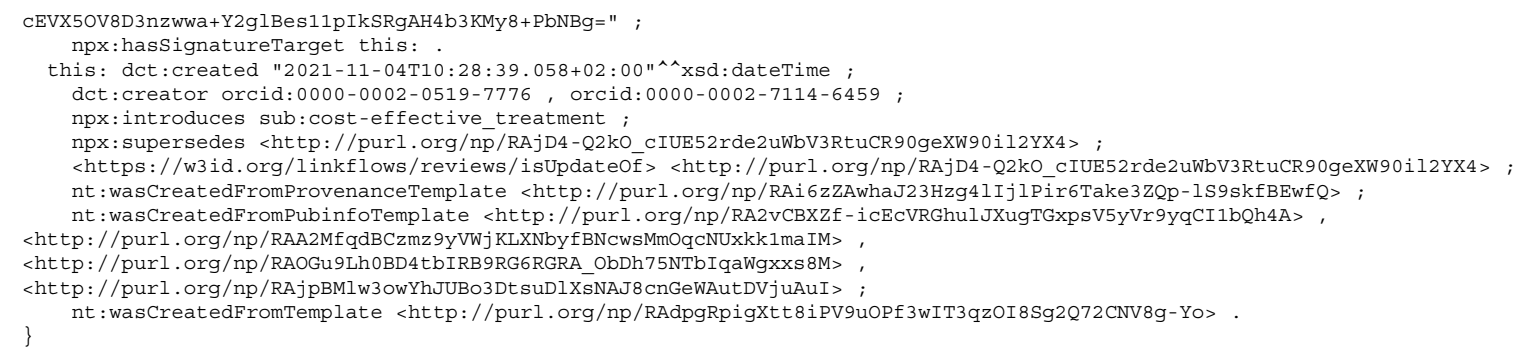

\section{References}

[1] C.I. Bucur, T. Kuhn, D. Ceolin and J. van Ossenbruggen, Expressing high-level scientific claims with formal semantics, in: Proceedings of the 11th Knowledge Capture Conference, 2021. doi:10.1145/3460210.3493561.

[2] V. Fragoulakis, M. Bartsakoulia, X. Díaz-Villamarín, K. Chalikiopoulou, K. Kehagia, J.G.S. Ramos, L.J. MartínezGonzález, M. Gkotsi, E. Katrali, E. Skoufas, A. Vozikis, A. John, B.R. Ali, S. Wordsworth, C.L. Dávila-Fajardo, T. Katsila, G.P. Patrinos and C. Mitropoulou, Cost-effectiveness analysis of pharmacogenomics-guided clopidogrel treatment in Spanish patients undergoing percutaneous coronary intervention, Pharmacogenomics J. 19(5) (2019), 438-445. doi:10.1038/ s41397-019-0069-1. 